\title{
Mechanical Parameters Identification for Laminated Composites Based on the Impulse Excitation Technique
}

\author{
Wenlei Song, Yongteng Zhong, Jiawei Xiang* \\ College of Mechanical \& Electrical Engineering, Wenzhou University, Wenzhou, 325035, \\ P.R. China. \\ Corresponding author: Jiawei Xiang, Professor \\ E-mail: wxw8627@163.com
}

\begin{abstract}
This study suggests a simple, quick and non-destructive method for investigation of mechanical parameters (dynamic Young's modulus, dynamic shear modulus and Poisson's ratio) detection for rectangular plate structures in laminated composites which only utilizes the fundamental resonant frequency in flexural and torsional modes, mass and dimensions of structures. The method is based on the impulse excitation technique (IET) to pick up the fundamental resonant frequency and then the corresponding formulas are applied to evaluate the mechanical parameters. Numerical simulations using finite element method (FEM) and experimental investigations of several cases based on IET are introduced to verify the accuracy of the IET formulas. The results show that the IET is applicable for mechanical parameters identification for laminated composites plates. The method is expected to detect mechanical parameters of other more complicated structures.
\end{abstract}

Keywords: impulse excitation technology; mechanical parameters; laminated composites.

\section{Introduction}

The IET method is a convenient and non-destructive testing method covers determination of the dynamic Young's modulus and dynamic shear modulus of elastic materials, which is based on the structures of these materials possess specific mechanical resonant frequencies that are determined by the elastic properties. The methods use the measurement of resonant frequency of structures for the purposes of dynamic mechanical parameters detection [1]. There are different strategies suggested for mechanical parameters detection purposes depending on the type of the mode parameters used: natural frequency, displacement signal, loading force, deflection [2-4]. All of these have their advantages and disadvantages. The natural frequencies are commonly used to detect the dynamic modulus of structures by comparison. The IET method is easy to perform and requires a very short time and nondestructive. In this technique, dynamic Young's modulus and dynamic torsional modulus can be calculated directly using the data of resonant frequency, dimensions and mass.

Ferreira et al. proposed some methods about analysis of structures in composites using the finite element method and Yang et al. developed approaches of damage detection for composites [5-16], it is essential to know the mechanical parameters of materials before establishing the FEM model [17-19]. However, the mechanical parameters can not be obtained accurately using theoretical method. Therefore, the IET methods for the measurement of dynamic Young's modulus and the dynamic shear modulus that are based on the resonant frequencies of a structure present a very attractive possibility since these are quite easy and convenient to obtain from experiments. The dynamic Young's modulus and dynamic shear modulus detection methods associated with resonant frequency measurement 
have drawn special attention in the literatures [20-24]. Generally, there are two procedures to accomplish the dynamic Young's modulus and dynamic shear modulus detection in structures. The first procedure is picking up the vibration signals of structures. The second procedure is analyzing the vibration signals and getting the resonant frequency in flexural and torsional modes. When the resonant frequency is known and the dynamic Young's modulus and dynamic shear modulus can be calculated using their relationship with resonant frequencies respectively. However, so far only the dynamic Young's modulus and dynamic shear modulus detection methods for simple structures (beams of rectangular cross section and rods of circular cross section) are well established and the calculation formulas of dynamic Young's modulus and dynamic shear modulus are also reported [25-26]. The reason is that the correction factors depend the shape of structures are difficult to obtain accurately. Thus some researches have been done about mechanical parameters detection in different materials [2737], environmental variability [38-45], different dimension sizes [46], etc., based on beams of rectangular cross section and rods of circular cross section only. Moreover, we try to do some exploration about mechanical parameters detection for laminated composites based on plates of rectangular cross section.

Because there is little research for indentifying the dynamic Young's modulus and dynamic shear modulus of laminated composites, the purpose of the present work is to do some numerical simulations and experimental investigations to prove the existing formulas based on IET are applicable to calculate the dynamic Young's modulus and dynamic shear modulus for laminated composites. With this method, Numerical models using FEM software ANSYS are built and some experimental investigations are performed. The simulation results are compared with literatures and the precision of the present method is examined. The performance of this method has also been examined using experimental data of a laminated plate. The detection result shows that the proposed method can be applied to real structures. The rest of the paper is organized as follows. The next section introduces the formula based on IET to calculate the dynamic Young's modulus, dynamic shear modulus and Poisson's ratio of structures. Numerical simulations and Experimental investigations are presented in sections 3 and 4, respectively.

\section{Technique methods}

The IET measures the fundamental resonant frequency of plates of rectangular cross section with suitable geometry by exciting them mechanically by an impact hammer. An accelerometer senses the resulting mechanical vibrations of plates and transforms them into electric signals. Support locations, impulse locations and signal pick-up points are selected to induce and measure specific modes of the transient vibrations. The signals are analyzed and the fundamental resonant frequency is isolated and measured by the signal analyzer, which provides a numerical reading that are the first-order flexural resonant frequency and the firstorder torsional resonant frequency of plates vibration. The appropriate fundamental resonant frequencies, dimensions, and mass of the specimen are used to calculate dynamic Young's modulus, dynamic shear modulus, and Poisson's ratio.

For the calculation of Young's modulus and shear modulus based on the theory of Pickett[25] have been employed, which describe the calculation of the Young's modulus and shear modulus from resonant frequency, dimensions, mass and Poisson's ratio of a rectangular solid.

Support the structures at the fundamental nodal points. The locations of fundamental nodal points are $0.224 L$ from each end for flexural modes and it should be the center point of structure for torsional mode, as shown in Fig. 1. 


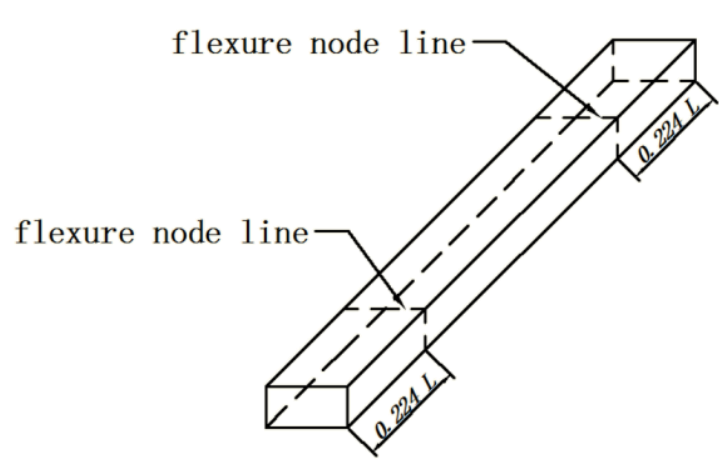

(a) The flexural modes

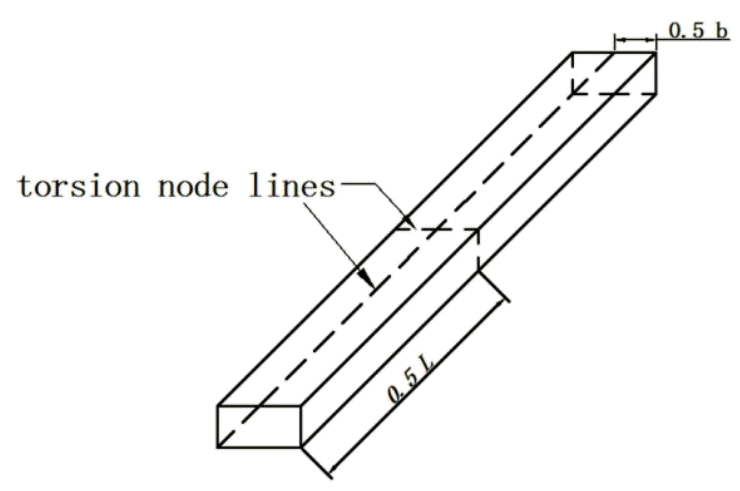

(b) The torsional modes

Fig. 1. The diagrammatic sketch of structures

By experimental modal analysis (EMA), the fundamental flexural resonant frequency and fundamental torsional resonant frequency will be obtained accordingly, and the Young's modulus and shear modulus will finally be calculated from the formulas, as shown in the following two sub-sections.

\subsection{The calculation of dynamic Young's modulus}

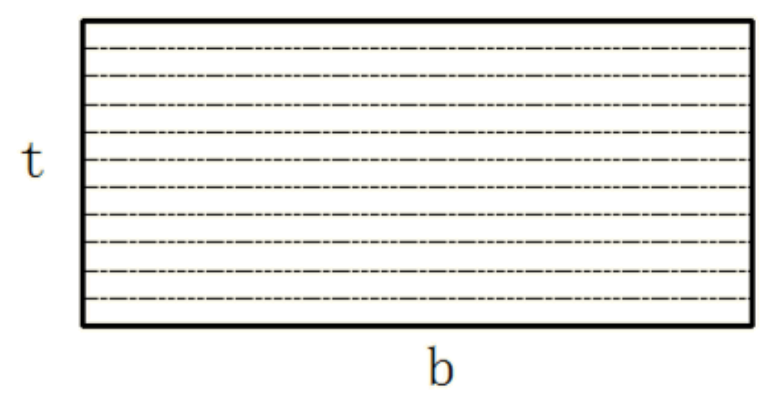

Fig. 2. The diagram of laminated composite plate cross-section

The diagram of plate cross-section is shown in Fig. 2. For a fundamental flexural vibration, the Young's modulus of plate can be expressed as [24]:

$$
E=0.9465\left(m f_{f}^{2} / b\right)\left(L^{3} / t^{3}\right) T_{1}
$$

where $E$ is the Young's modulus $(P a), m$ is mass $(g), b$ is width $(m m), L$ is length $(\mathrm{mm}), t$ is thickness $(\mathrm{mm}), f_{f}$ is the fundamental flexural resonant frequency $(\mathrm{Hz}), T_{1}$ is correction factor for fundamental flexural mode to account for the thickness of the plates, which is calculated by

$$
T_{1}=1+6.585\left(1+0.0752 \mu+0.8109 \mu^{2}\right)(t / L)^{2}-0.868(t / L)^{4}-\left[\frac{8.340\left(1+0.2023 \mu+2.173 \mu^{2}\right)(L / t)^{4}}{1.000+6.338\left(1+1.1408 \mu+1.536 \mu^{2}\right)(L / t)^{2}}\right]
$$

where $\mu$ is Poisson's ratio. To simple the calculation, if $L / t \geq 20, T_{1}$ can be simplified as

$$
T_{1}=1.000+6.585(L / t)^{2}
$$

and $E$ can be directly obtained using Eq.(1).

If $L / t \leq 20$ and $\mu$ is known, $T_{1}$ can be calculated using Eq. (2). Then $E$ can also be directly obtained using Eq. (1). 
If $L / t \leq 20$ and $\mu$ is not known, then we must assume an initial Poisson's ratio and use an iterative process to determine a value of Poisson's ratio. The iterative process is shown in Fig.3.

\subsection{The calculation of dynamic shear modulus}

For the fundamental torsion frequency of a plate, the shear modulus $G$ can be calculated as follow [24]:

$$
G=\frac{4 L m f_{t}^{2}}{b t}[B /(1+A)]
$$

where $G$ is dynamic shear modulus $(P a), f_{t}$ is fundamental torsional resonant frequency of the plate $(H z) . A$ and $B$ are the empirical correction factor dependent on the width-to-thickness.

$$
B=\left[\frac{b / t+t / b}{4(t / b)-2.52(t / b)^{2}+0.21(t / b)^{6}}\right]
$$

and

$$
A=\left[\frac{\left[0.5062-0.8776(b / t)+0.3504(b / t)^{2}-0.0078(b / t)^{3}\right]}{\left[12.03(b / t)+9.892(b / t)^{2}\right]}\right]
$$

\subsection{The calculation of Poisson's ratio}

The Poisson's ratio could be calculated as

$$
\mu=\frac{E}{2 G}-1
$$

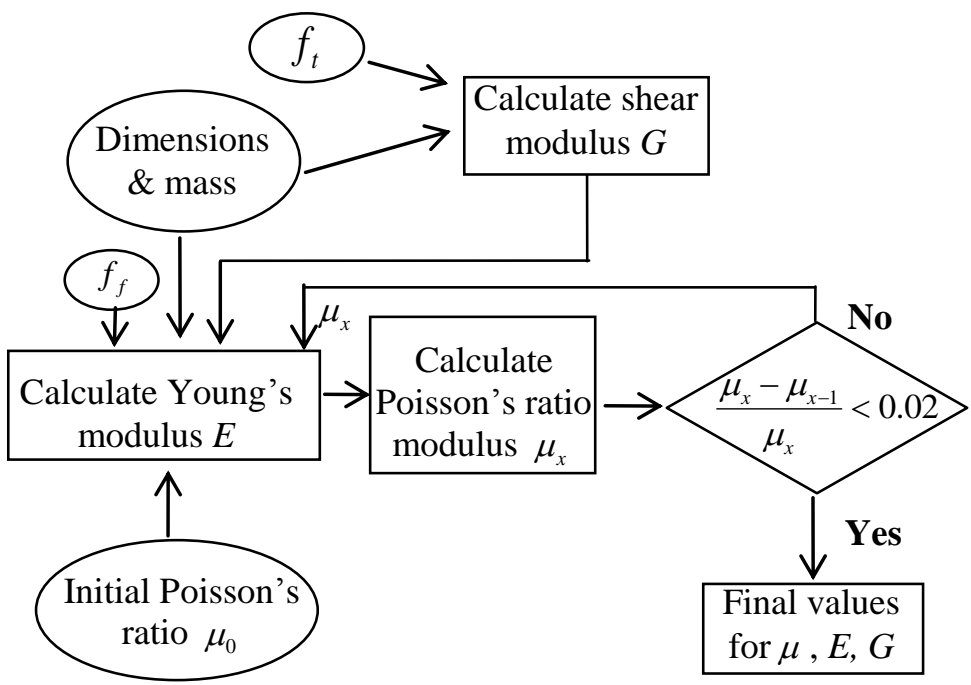

Fig. 3. The flow diagram of iterative process

The procedure of iterative process can be described as follow, firstly, using the Eqs mentioned above, calculate the dynamic Young's modulus $E$ from the fundamental flexural frequency $f_{f}$ and the initial Poisson's ratio $\mu_{0}$, and calculate the dynamic shear modulus $G$ from the fundamental torsional frequency $f_{t}$. Secondly, substitute $E$ and $G$ into the Eq. (7) and calculate a new value of Poisson's ratio for anther iteration beginning. Repeat the iterative process until no significant difference (0.02 or less) is observe between $\mu_{x}$ and $\mu_{x-1}$. 


\section{Simulation procedures}

To prove the feasibility of IET method and the accuracy of formulas for calculating the mechanical parameters of plates in laminated composites. Numerical simulations are applied using commercial finite element (FE) software ANSYS.

Table 1 shows the geometry dimensions of structures. "Solid 191" element was applied in ANSYS program to conduct simulation of plates in laminated composites (set parameters as: $\left.E^{*}=24 G P a, \mu=0.3, \rho=1800 \mathrm{~kg} / \mathrm{m}^{3}\right)$. The structures' support locations are located in flexure node lines and torsion node lines shown in Fig. 1(a) and Fig. 1(b), respectively.

Table 1 The dimension of structures

\begin{tabular}{cccc}
\hline case & Length $(m)$ & Width $(m)$ & $\begin{array}{c}\text { Thickness } \\
(m)\end{array}$ \\
\hline 1 & 0.15 & 0.01 & 0.005 \\
2 & 0.20 & 0.01 & 0.005 \\
3 & 0.15 & 0.02 & 0.005 \\
4 & 0.15 & 0.01 & 0.006 \\
5 & 0.20 & 0.02 & 0.006 \\
\hline
\end{tabular}

Modal analysis is performed to get the resonant frequency in flexural modes and torsional modes. According to the computation, the first-order flexural resonant frequency and firstorder torsional resonant frequency can be obtained (recorded in Table 2).

Table 2 The first-order flexural frequency and first-order torsional frequency for structures

\begin{tabular}{cccccc}
\hline case & 1 & 2 & 3 & 4 & 5 \\
\hline$f_{f}(H z)$ & 831.071 & 468.247 & 832.976 & 995.444 & 562.016 \\
$f_{t}(H z)$ & 5593.8 & 4194.9 & 3370.8 & 6145.7 & 2934.4 \\
\hline
\end{tabular}

When the first-order flexural frequency $f_{f}$ and first-order torsional frequency $f_{t}$ for plates is known, the Young's modulus E can be calculated using Eq.(1) and Eq.(2), and the shear modulus $G$ will be obtained using Eqs.(4-6). Besides, the absolute relative errors of the dynamic Young's modulus $\varepsilon_{E}$ and dynamic shear modulus $\varepsilon_{G}$ between the measured and theoretical values can be calculated using Eq.(9) and Eq.(10), respectively. The results are shown in Table 3.

$$
\begin{aligned}
& \varepsilon_{E}=\frac{\left|E-E^{*}\right|}{E^{*}} \times 100 \\
& \varepsilon_{G}=\frac{\left|G-G^{*}\right|}{G^{*}} \times 100
\end{aligned}
$$

Where $G^{*}$ is the theoretical value of shear modulus $G^{*}=\frac{E^{*}}{2(1+\mu)}$. 
Table 3 The calculation results of Young's modulus and shear modulus for structures

\begin{tabular}{cccccc}
\hline case & 1 & 2 & 3 & 4 & 5 \\
\hline$E(G P a)$ & 24.017 & 24.013 & 24.127 & 24.012 & 24.070 \\
$\mathcal{E}_{E}(\%)$ & 0.073 & 0.055 & 0.533 & 0.051 & 0.294 \\
$G(G P a)$ & 9.214 & 9.212 & 9.191 & 9.233 & 9.184 \\
$\mathcal{E}_{G}^{(\%)}$ & 0.171 & 0.192 & 0.428 & 0.032 & 0.503 \\
\hline
\end{tabular}

As shown in Table 3, the relative errors $\varepsilon_{E}$ for the dynamic Young's modulus are varying from $0.051 \%$ to $0.294 \%$, whereas the errors are changing from $0.032 \%$ to $0.428 \%$ for the relative errors of shear modulus $\varepsilon_{G}$.

The above numerical simulations clearly demonstrates that the mechanical parameters detection using the standard IET, the FEM simulation for plates in laminated composites is efficient.

\section{Experimental investigations}

\subsection{Experimental procedure}

This test method measures the fundamental resonant frequency of plates in laminated composites by exciting them mechanically by an impact-hammer. A contact accelerometer senses the resulting mechanical vibration of plates. The signals are performed fast Fourier transform (FFT) analysis and the fundamental resonant frequency is isolated and measured by the signal analyzer (AVANT MI-7016 and computer) which provides a numerical reading. The appropriate fundamental resonant frequencies, dimensions, and mass of plates are used to calculate dynamic Young's modulus, dynamic shear modulus, and Poisson's ratio.

The dimensions of composite structures (epoxy plates) using in experiments is shown in Table 4.

Table 4 The dimensions of plates in experiments

\begin{tabular}{cccc}
\hline case & 1 & 2 & 3 \\
\hline Length $(m)$ & 0.300 & 0.400 & 0.600 \\
Width $(m)$ & 0.200 & 0.400 & 0.300 \\
thickness $(m)$ & 0.006 & 0.006 & 0.006 \\
\hline
\end{tabular}

\subsection{Fundamental flexural resonant frequency}

The plates are hanged on flexure node lines by elastic wires. Place the accelerometer in contact with plates to pick up the desired vibration.

Strike the plates lightly and elastically, either at the center of them or at the opposite end of them from the detecting accelerometer. 
Record the resultant reading, and repeat the test until five consecutive readings are obtained that lie within $1 \%$ of each other. The average of these five records is then applied to determine the fundamental resonant frequency in flexure.

The experimental setups in flexural mode are shown in Fig. 4

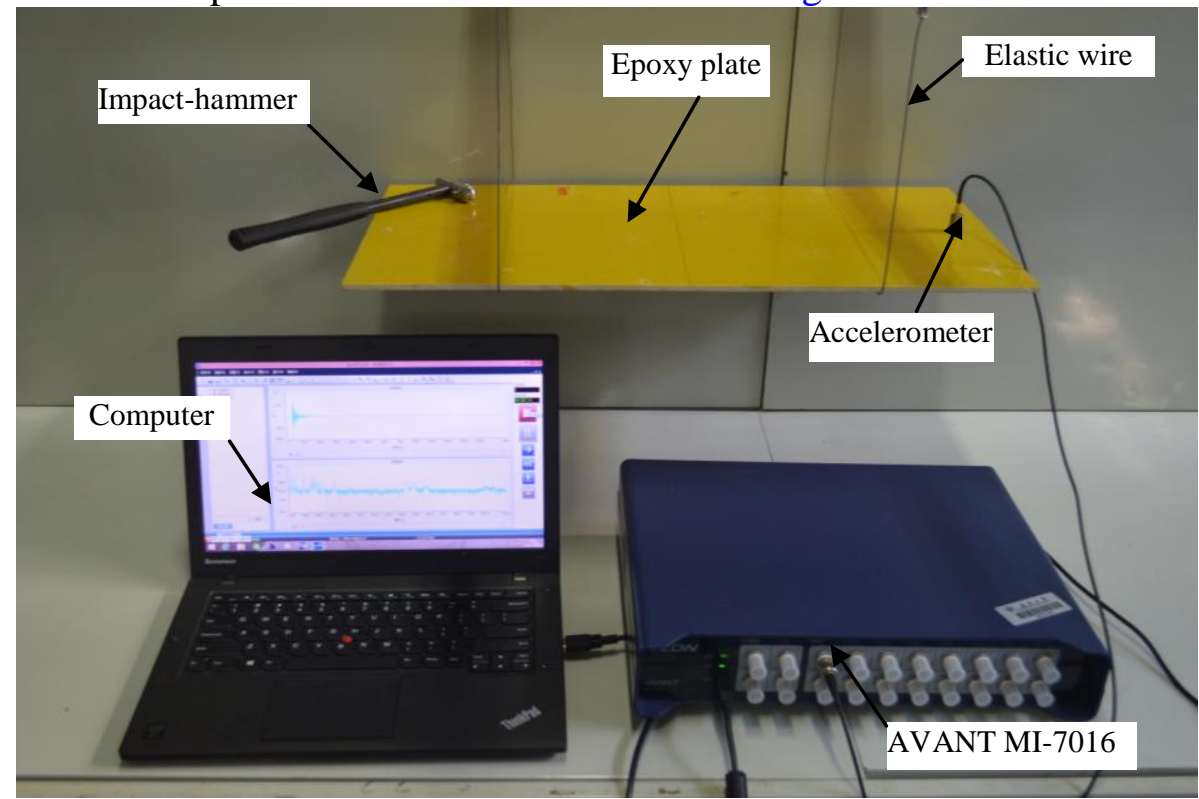

Fig. 4. Photographs of the experimental test setup in flexural mode

The measured impulse response signal and the corresponding frequency spectrum of plates in flexural mode are shown in Fig. 5.

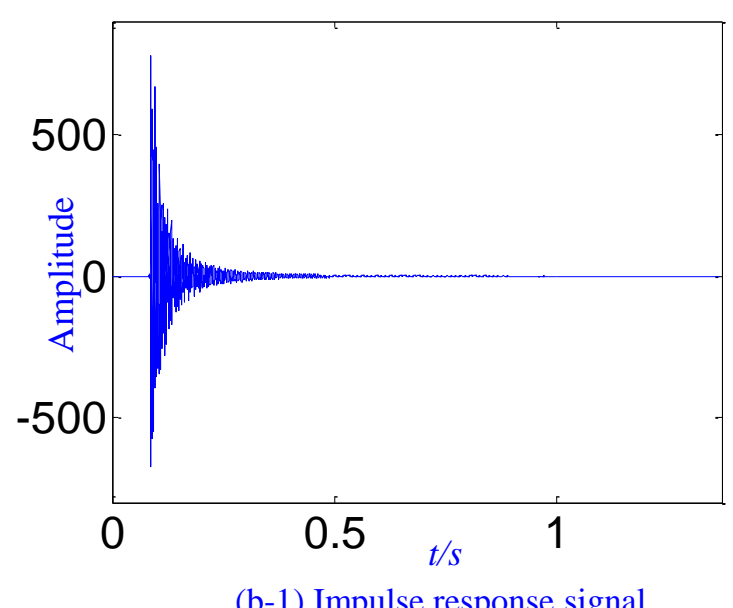

(b-1) Impulse response signal



(b-2) Frequency spectrum

(a) Case 1 


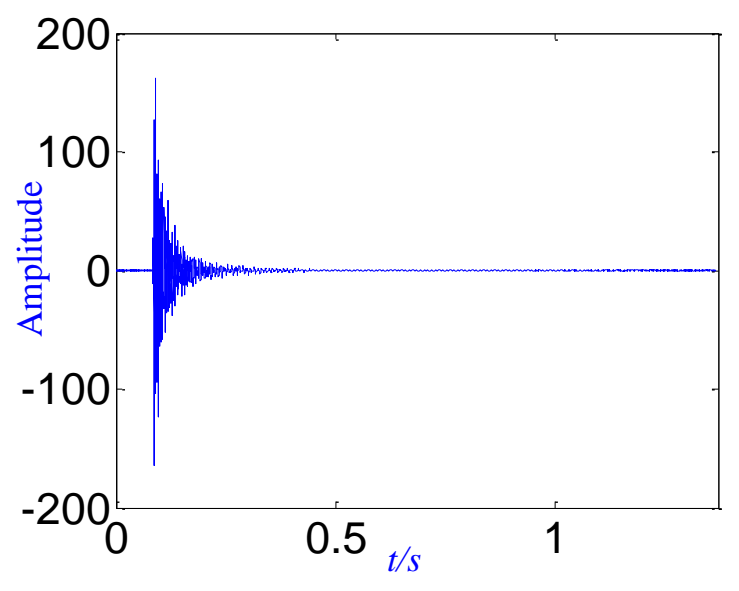

(b-1) Impulse response signal

(b) Case 2

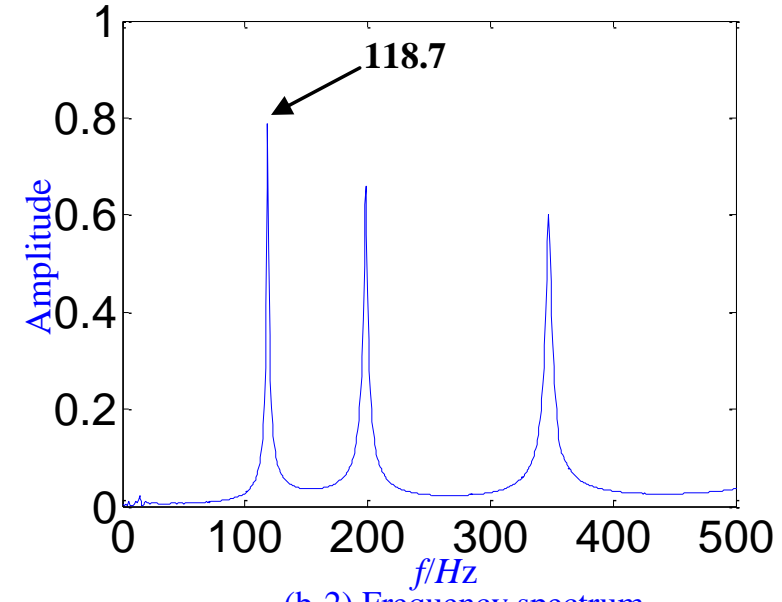

(b-2) Frequency spectrum

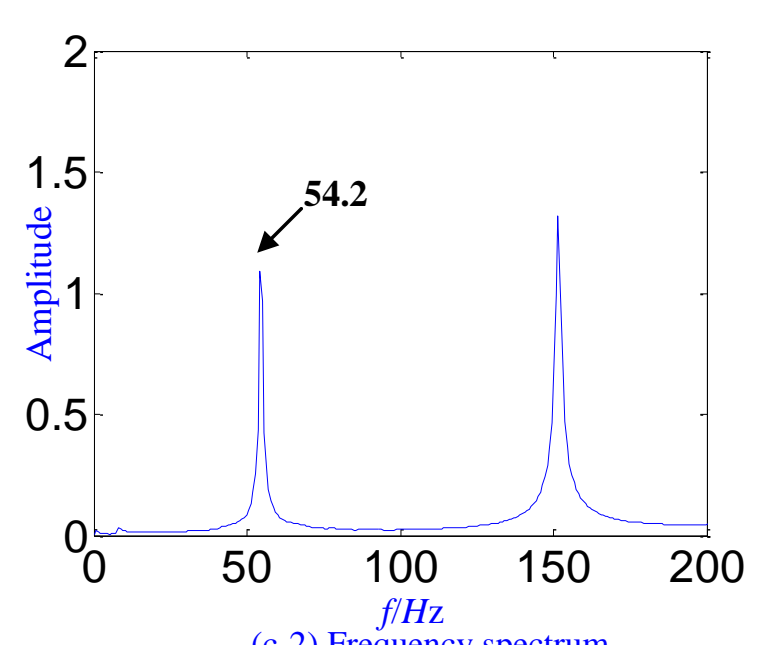

(c-1) Impulse response signal



(c) Case 3

Fig. 5. The measured impulse response signal and the corresponding frequency spectrum in flexural mode

\subsection{Fundamental torsional resonant frequency}

Hanging the plates at the midpoint of their length and width (the torsion node lines, see Figure 1(b)), we locate the accelerometer at one quadrant of the plate, preferably at approximately $0.224 \mathrm{~L}$ from one end and towards the edge. This location is a nodal point of flexural vibration and will minimize the possibility of detecting a spurious flexural mode.

Strike the plates on the quadrant diagonally opposite the accelerometer, again at $0.224 \mathrm{~L}$ from the end and near the edge. Strike at a flexural nodal point will minimize the possibility of exciting a flexural mode of vibration.

Record the resultant reading, and repeat the test until five consecutive readings are obtained that lie within $1 \%$ of each other. Similarly, the average of these five records is then applied to determine the fundamental resonant frequency in torsion.

The experimental apparatus for plate in torsional mode is shown in Fig. 6. 


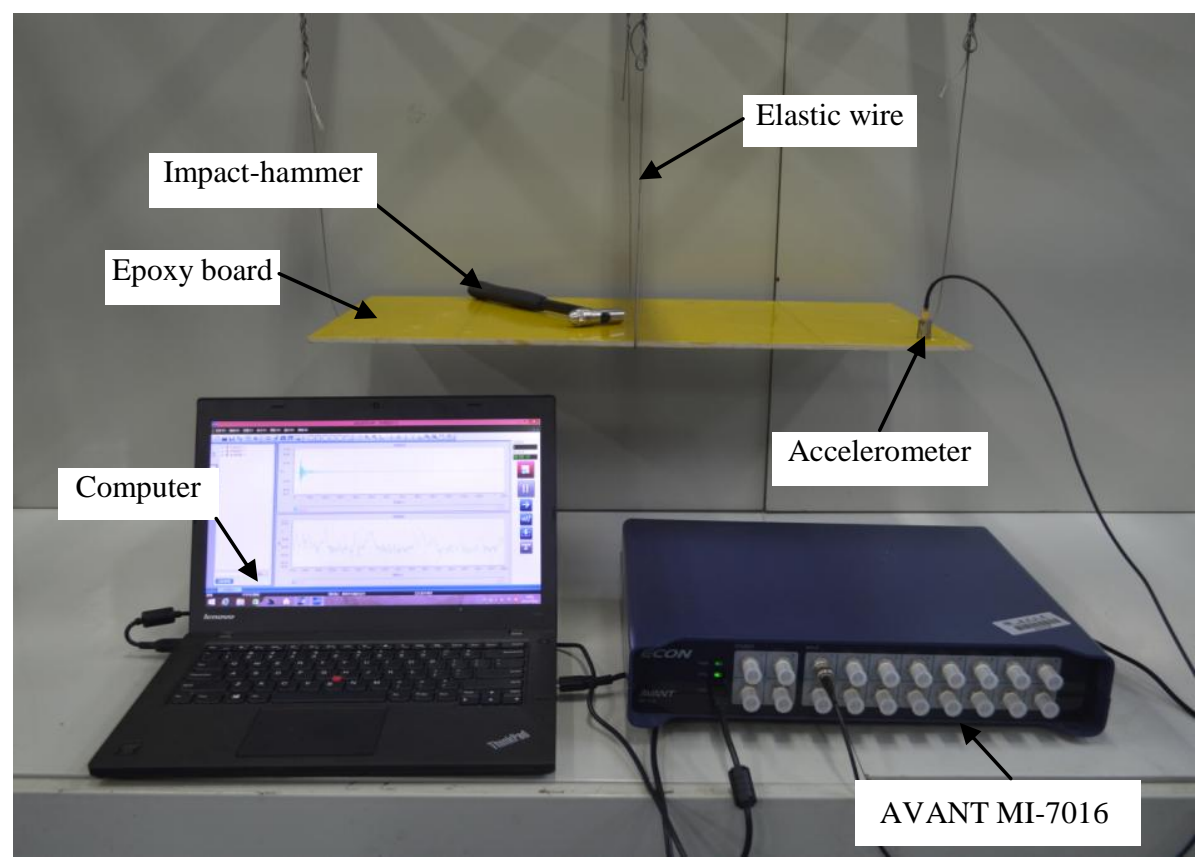

Fig. 6. Photographs of the experimental test setup in torsional mode

The measured impulse response signal and the corresponding frequency spectrum of plates in torsional mode are shown in Fig. 7.

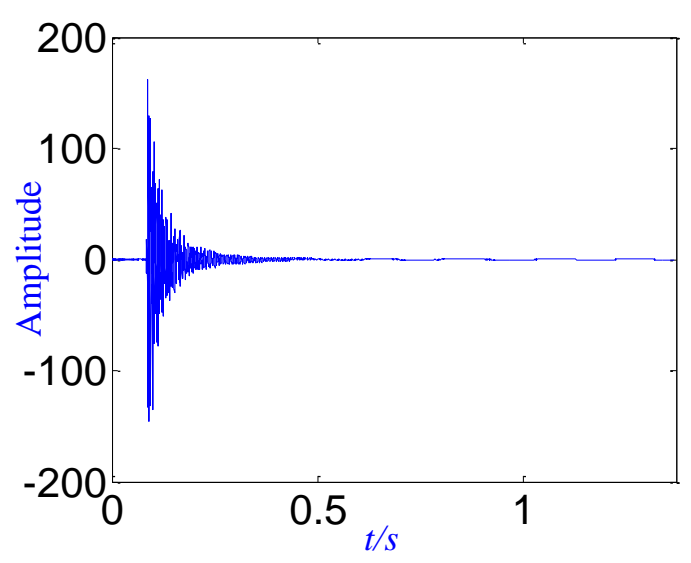

(a-1) Impulse response signal

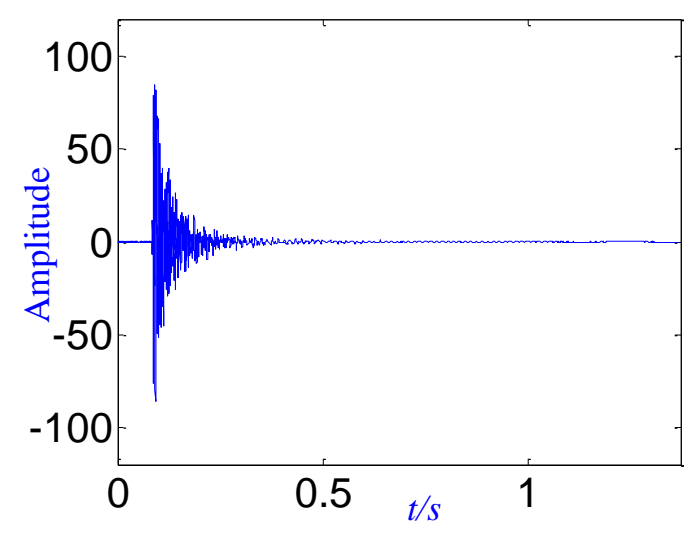

(b-1) Impulse response signal

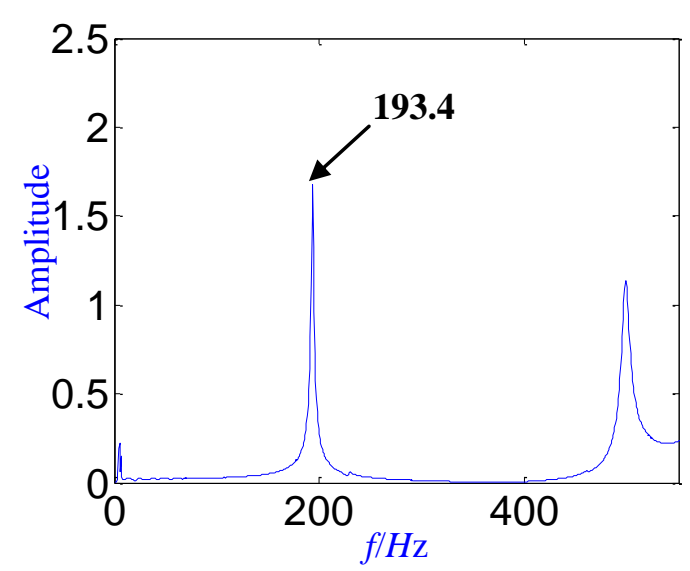

(a-2) Frequency spectrum

(a) Case 1

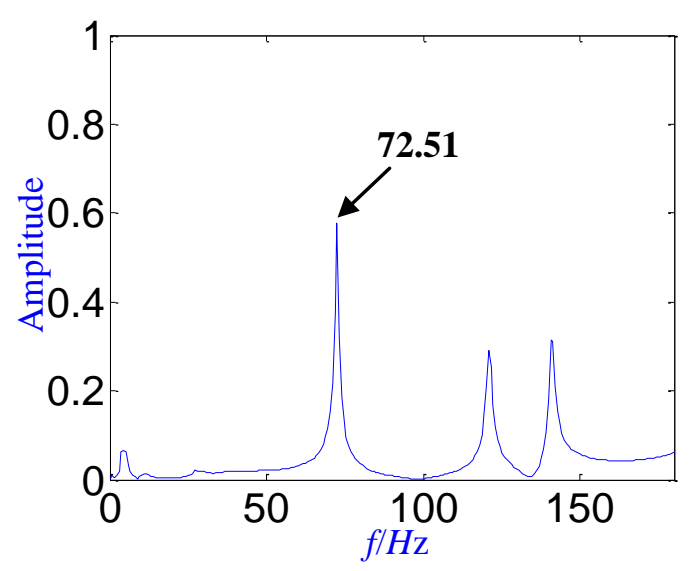

(b-2) Frequency spectrum

(b) Case 2 


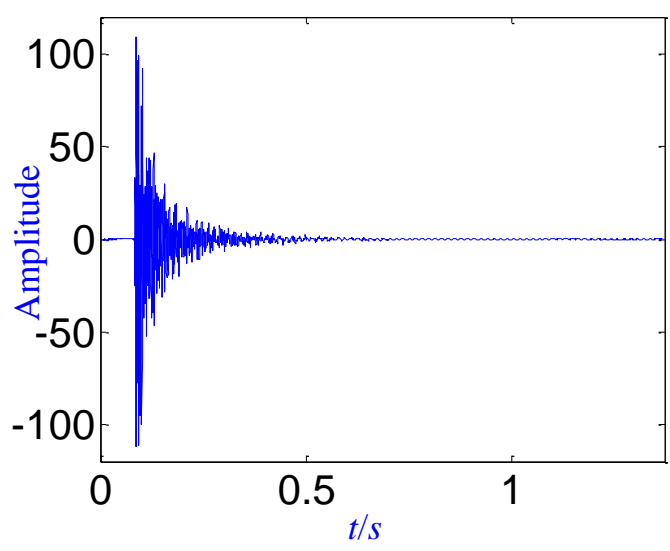

(c-1) Impulse response signal

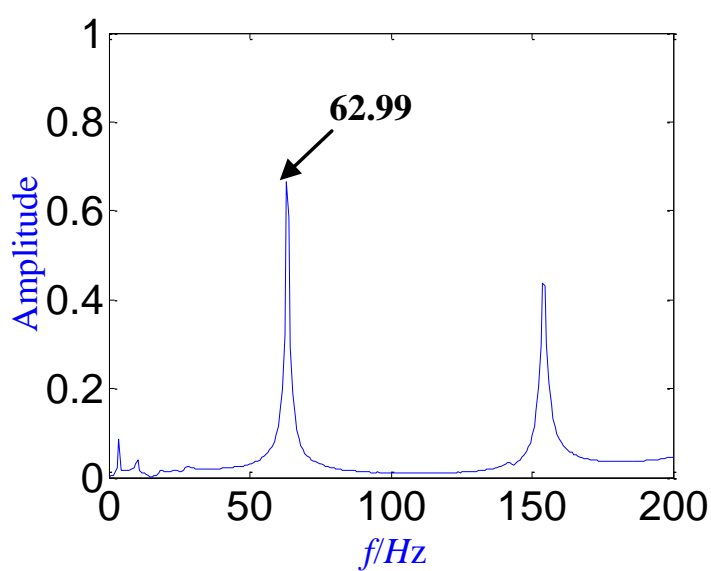

(c-2) Frequency spectrum

(c) Case 3

Fig. 7. The measured impulse response signal and the corresponding frequency spectrum in torsional mode

The measured first-order flexural resonant frequencies $f_{f}$ and the first-order torsional resonant frequencies $f_{t}$ for plate (three Cases) are shown in Table5.

Similar to the numerical simulation in Section 3, when the first-order resonant frequency and the first-order resonant frequency $\left(f_{f}\right.$ and $f_{t}$ ) for plate are measured, Young's modulus $E$ and shear modulus $G$ will be calculated. Table 5 also gives the absolute relative errors of the mechanical parameters $\left(\varepsilon_{E}\right.$ and $\varepsilon_{G}$ ) between the measured and theoretical values can be calculated using Eq. (9) and (10), respectively.

Table 5 The experimental results

\begin{tabular}{cccc}
\hline case & 1 & 2 & 3 \\
\hline$f_{f}(H z)$ & 221.9 & 118.7 & 54.2 \\
$f_{t}(H z)$ & 193.4 & 72.51 & 62.99 \\
$E(G P a)$ & 18.394 & 18.754 & 18.031 \\
$\varepsilon_{E}(\%)$ & 0.005 & 1.96 & 1.97 \\
$G(G P a)$ & 6.825 & 6.921 & 6.549 \\
$\varepsilon_{G}(\%)$ & 0.886 & 2.305 & 3.19 \\
$\mu$ & 0.3475 & 0.3548 & 0.3700 \\
$\varepsilon_{\mu}(\%)$ & 2.77 & 0.727 & 3.52 \\
\hline
\end{tabular}

For experimental investigations, Table 5 show the relative errors of dynamic Young's modulus $\varepsilon_{E}$ are varying from $0.005 \%$ to $1.97 \%$, and the relative errors of dynamic shear modulus $\varepsilon_{G}$ are varying from $0.886 \%$ to $3.19 \%$.However, the errors $\varepsilon_{\mu}$ are changing from $0.727 \%$ to $3.52 \%$ for the detection of the Poisson's ratio $\mu$.

The above experiment investigations clearly demonstrates that the mechanical parameters detection using the standard IET, the FEM simulation can be applied to plates in laminated composites. 


\section{Conclusions}

This paper suggests a simple procedure for the dynamic Young's modulus and dynamic shear modulus detection in laminated composites for rectangular plate structures which is based on the impulse excitation technique (IET). The procedure involves two steps. The first step is the acquisition of vibration signal in flexural mode and torsional mode for laminated composites plates. The second procedure is analyzing the vibration signals and getting the first-order flexural resonant frequency and the first-order torsional resonant frequency. Then the values of frequencies are submitted into the existing formulas to calculate the dynamic Young's modulus and dynamic the shear modulus of laminated composites, respectively.

In the present method, we use numerical simulations and experimental investigations to verify the accuracy of the IET formulas. In the simulation, relative errors for the predicted dynamic Young's modulus are varying from $0.051 \%$ to $0.294 \%$, whereas the errors are changing from $0.032 \%$ to $0.428 \%$ for the relative errors predicted shear modulus. In the experimental investigations, they are a little bit increasing from $0.005 \%$ to $1.97 \%, 0.886 \%$ to $3.19 \%$ and $0.727 \%$ to $3.52 \%$, respectively. The results show that the IET is applicable for mechanical parameters identification for laminated composites. The basic idea of this method is expected to be developed for more complicated structures using numerical simulation based experimental design technique, such as surface response method, orthogonal experiment design, etc.

\section{Acknowledgments}

The authors are grateful to the support from the National Science Foundation of China (Nos. 51575400, 51505339, 51405346), the Zhejiang Provincial Natural Science Foundation of China (Nos.LR13E050002, LQ161050005), the Zhejiang Technologies R\&D Program of China (No. 2014C31103).

\section{References}

[1] Alfano M, Pagnotta L. A non-destructive technique for the elastic characterization of thin isotropic plates. Ndt \& E International 2007; 40: 112-20.

[2] Zeng X, Wen S, Li M. Estimating Young's modulus of materials by a new three-point bending method. Adv Mater Sci Eng 2014; 2014.

[3] Zhang E, Chazot JD, Antoni J. Bayesian characterization of Young's modulus of viscoelastic materials in laminated structures. J Sound Vib 2013; 332: 3654-66.

[4] Sousa FJP, Dal Bó M, Guglielmi PO. Characterization of Young's modulus and fracture toughness of albite glass by different techniques. Ceram Int 2014; 40: 10893-99.

[5] Belinha J, Araújo AL, Ferreira AJM, Dinis LMJS, Jorge RN. The analysis of laminated plates using distinct advanced discretization meshless techniques. Compos Struct 2016; 143: $165-79$.

[6] Liu B, Ferreira AJM, Xing YF, Neves AMA. Analysis of functionally graded sandwich and laminated shells using a layerwise theory and a differential quadrature finite element method. Compos Struct 2016; 136: 546-53.

[7] Liu B, Xing YF, Eisenberger M, Ferreira AJM. Thickness-shear vibration analysis of rectangular quartz plates by a numerical extended Kantorovich method. Compos Struct 2014; 107: 429-35.

[8] Natarajan S, Ferreira AJM, Bordas SPA, Carrera E, Cinefra M. Analysis of composite plates by a unified formulation-cell based smoothed finite element method and field consistent elements. Compos Struct 2013; 105: 75-81.

[9] Thai CH, Ferreira AJM, Carrera E, Nguyen-Xuan H. Isogeometric analysis of laminated composite and sandwich plates using a layerwise deformation theory. Compos Struct 
2013; 104: 196-214.

[10] Roque CMC, Fidalgo DS, Ferreira AJM, Reddy JN. A study of a microstructuredependent composite laminated Timoshenko beam using a modified couple stress theory and a meshless method. Compos Struct 2013; 96: 532-7.

[11] Castro LMS, Ferreira AJM, Bertoluzza S. A wavelet collocation method for the static analysis of sandwich plates using a layerwise theory. Compos struct 2010; 92(8): 178692.

[12] Ferreira AJM, Castro LMS, Bertoluzza S. A high order collocation method for the static and vibration analysis of composite plates using a first-order theory. Compos Struct 2009; 89(3): 424-32.

[13] Roque C M C, Ferreira A J M. New developments in the radial basis functions analysis of composite shells. Compos Struct 2009; 87(2): 141-50.

[14] Yang Z B, Radzienski M, Kudela P, Ostachowicz W. Scale-wavenumber domain filtering method for curvature modal damage detection. Compos Struct 2016; 154: 396409.

[15] Yang Z B, Radzienski M, Kudela P, Ostachowicz W. Two-dimensional modal curvature estimation via Fourier spectral method for damage detection. Compos Struct 2016; 148 : 155-67.

[16] Zuo H, Yang Z, Chen X, Xie Y, Miao H. Analysis of laminated composite plates using wavelet finite element method and higher-order plate theory. Compos Struct 2015; 131: 248-58.

[17] Yang Z, Chen X, Li X, Jiang Y. Miao H, He Z. Wave motion analysis in arch structures via wavelet finite element method. J Sound Vib 2014; 333(2): 446-469.

[18] Zhang X, Gao R X, Yan R, Chen X, Sun C, Yang Z. Multivariable wavelet finite element-based vibration model for quantitative crack identification by using particle swarm optimization. J Sound Vib 2016; 375: 200-216.

[19] Zhang X W, Zuo H, Liu J X, Chen X F, Yang Z.B. Analysis of shallow hyperbolic shell by different kinds of wavelet elements based on B-spline wavelet on the interval. App Math Model 2016; 40(3): 1914-1928.

[20] Spinner S, Reichard TW, Tefft WE. A comparison of experimental and theoretical relations between Young's modulus and the flexural and longitudinal resonance frequencies of uniform bars. J Res Natl Bur Stand Phys Chem 1960; 64: 147-55.

[21] Wang Z., Guo Z., Guo L., Liu B., Wang Y., Yang Y. Torsional vibration shape method of free plate for testing shear modulus of lumber, Sci Silvae Sin 2014; 50: 122-8.

[22] Tefft W E, Spinner S. Torsional resonance vibrations of uniform bars of square cross section. J Res Natl Bur Stand Phys Chem 1961; 65: 167-71.

[23] Timoshenko S. Mechanical Vibration Science, China Machine Press, Beijing, 1965.

[24] ASTM E. Standard test method for dynamic Young's modulus, shear modulus, and Poisson's ratio by sonic resonance. Annual Book of ASTM Standards, 2001.

[25] Pickett G. Equations for computing elastic constants from flexural and torsional resonant frequencies of vibration of prisms and cylinders, 1900.

[26] De Oliveira APN, Vilches ES, Soler VC, Villegas FAG. Relationship between Young's modulus and temperature in porcelain tiles. J Eur Ceram Soc 2012; 32: 2853-8.

[27] Hauert A, Rossoll A, Mortensen A. Young's modulus of ceramic particle reinforced aluminium: Measurement by the Impulse Excitation Technique and confrontation with analytical models. Compos Part A-Appl S 2009; 40: 524-9.

[28] Bahr O, Schaumann P, Bollen B. Young's modulus and Poisson's ratio of concrete at high temperatures: Experimental investigations. Mater Design 2013; 45: 421-9.

[29] Chiu CC, Case ED. Elastic modulus determination of coating layers as applied to layered ceramic composites. Mater Sci Eng A 1991; 132: 39-47. 
[30] Lugovy M, Slyunyayev V, Orlovskaya N, et al. Temperature dependence of elastic properties of ZrB 2-SiC composites. Ceram Int 2016; 42: 2439-45.

[31] Popovics JS, Kolluru SV, Shah SP. Determining elastic properties of concrete using vibrational resonance frequencies of standard test cylinders. Cement Concrete Aggr 2000; 22: 81-9.

[32] Roebben G, Van der Biest O. Recent advances in the use of the impulse excitation technique for the characterisation of stiffness and damping of ceramics, ceramic coatings and ceramic laminates at elevated temperature. Key Engineering Materials. Trans Tech Publications 2002; 206: 621-4.

[33] Schmidt R, Wicher V, Tilgner R. Young's modulus of moulding compounds measured with a resonance method. Polym test 2005; 24: 197-203.

[34] Tognana S, Salgueiro W, Somoza A. Measurement of the Young's modulus in particulate epoxy composites using the impulse excitation technique. Mater Sci Eng, A 2010; 527: 4619-23.

[35] Swarnakar AK, Donzel L, Vleugels J. High temperature properties of $\mathrm{ZnO}$ ceramics studied by the impulse excitation technique. J Eur Ceram Soc 2009; 29: 2991-8.

[36] Zhang E, Chazot JD, Antoni J. Bayesian characterization of Young's modulus of viscoelastic materials in laminated structures. J Sound Vib 2013; 332: 3654-66.

[37] Rupitsch SJ, Ilg J, Sutor A. Simulation based estimation of dynamic mechanical properties for viscoelastic materials used for vocal fold models. J Sound Vib 2011; 330: 4447-59.

[38] Latella BA, Liu T. High-temperature Young's modulus of alumina during sintering. J Am Ceram Soc 2005; 88: 773-6.

[39] Dickson RW, Schreiber E. An alumina standard reference material for resonance frequency and dynamic elastic moduli measurement II. Characteristic values for use from $250{ }^{\circ} \mathrm{C}$ to $10000{ }^{\circ} \mathrm{C}$. J Res. Natl Bur Stand Phys Chem 1973; 77: 391-4.

[40] Dickson RW, Wachtman JB. An alumina standard reference material for resonance frequency and dynamic elastic moduli measurement I. For use at $25{ }^{\circ} \mathrm{C}$. J Res Natl Bur Stand Phys Chem 1971; 75: 155-62.

[41] Pabst W, Gregorová E, Kloužek J. High-temperature Young's moduli and dilatation behavior of silica refractories. J Eur Ceram Soc 2016; 36: 209-20.

[42] Roebben G, Bollen B, Brebels A. Impulse excitation apparatus to measure resonant frequencies, elastic moduli, and internal friction at room and high temperature. Rev Sci Iistrm 1997; 68: 4511-5.

[43] Bahr O, Schaumann P, Bollen B. Young's modulus and Poisson's ratio of concrete at high temperatures: Experimental investigations. Mater Design 2013; 45: 421-9.

[44] Guillot FM, Trivett DH. Complete elastic characterization of viscoelastic materials by dynamic measurements of the complex bulk and Young's moduli as a function of temperature and hydrostatic pressure. J Sound Vib 2011; 330: 3334-51.

[45] Behmanesh I, Moaveni B. Accounting for environmental variability, modeling errors, and parameter estimation uncertainties in structural identification. J Sound Vib 2016; 374: 92-110.

[46] Wei CG, Bao YW, Liu ZQ. Influence of specimen size on the impulse excitation technique. Key Engineering Materials. Trans Tech Publications 2015; 633: 459-62. 